\title{
Return to play after treating acute muscle injuries in elite football players with radial extracorporeal shock wave therapy
}

\author{
James P. M. Morgan ${ }^{1}$, Mario Hamm², Christoph Schmitz ${ }^{1 *}$ (10 and Matthias H. Brem³
}

\begin{abstract}
Background: To compare lay-off times achieved by treating acute muscle injuries in elite football players with a multimodal therapy approach that includes a specific protocol of almost daily radial extracorporeal shock wave therapy (rESWT) with corresponding data reported in the literature.

Methods: We performed a retrospective analysis of treatments and recovery times of muscle injuries suffered by the players of an elite football team competing in the first/second German Bundesliga during one of the previous seasons.

Results: A total of 20 acute muscle injuries were diagnosed and treated in the aforementioned season, of which eight (40\%) were diagnosed as Type 1a/muscular tightness injuries, five (25\%) as Type $2 \mathrm{~b} /$ muscle strain injuries, four (20\%) as Type 3a/partial muscle tear injuries and three (15\%) as contusions. All injuries were treated with the previously mentioned multimodal therapy approach. Compared with data reported by Ekstrand et al. (Br J Sports Med 47:769-774, 2013), lay-off times (median/mean) were shortened by $54 \%$ and $58 \%$, respectively, in the case of Type 1 a injuries, by $50 \%$ and $55 \%$, respectively, in the case of Type $2 \mathrm{~b}$ injuries as well as by $8 \%$ and $21 \%$, respectively, in the case of Type 3a injuries. No adverse reactions were observed.
\end{abstract}

Conclusions: Overall, the multimodal therapy approach investigated in this study is a safe and effective treatment approach for treating Type $1 \mathrm{a}$ and $2 \mathrm{~b}$ acute muscle injuries amongst elite football players and may help to prevent more severe, structural muscle injuries.

Keywords: Acute muscle injury, Athletes, Extracorporeal shock wave therapy, Rehabilitation, Return-to-play

\section{Background}

Muscle injuries are the most common injury in football $[1,2]$. Non-structural injuries can contribute to over $50 \%$ of missed training sessions and participation in sporting activities and may worsen to become structural injuries if not appropriately managed [3]. According to MüllerWohlfahrt et al. [4], muscle injuries can be classified

\footnotetext{
*Correspondence: christoph_schmitz@med.uni-muenchen.de ${ }^{1}$ Chair of Neuroanatomy, Institute of Anatomy, Faculty of Medicine, Extracorporeal Shock Wave Research Unit, LMU Munich, Munich, Germany

Full list of author information is available at the end of the article
}

into indirect muscle injuries (comprising functional and structural injuries) and direct injuries (contusion, laceration) (Table 1). Some authors proposed to classify functional injuries as ultrastructural injuries [5]. Further classification of structural muscle injuries involves definition of the exact site of the lesion within the musculotendinous unit ranging from proximal $(\mathrm{P})$, middle $(\mathrm{M})$ and distal (D) [3].

Recent approaches to improve therapy for acute muscle injuries have mainly focused on Type 3b injuries (structural injuries involving significant muscle tears) $[6,7]$. In contrast, the treatment of Type $1 \mathrm{a}$ and $2 \mathrm{~b}$ functional/ ultrastructural muscle injuries as well as of Type $3 \mathrm{a}$ original author(s) and the source, provide a link to the Creative Commons licence, and indicate if changes were made. The images or other third party material in this article are included in the article's Creative Commons licence, unless indicated otherwise in a credit line to the material. If material is not included in the article's Creative Commons licence and your intended use is not permitted by statutory regulation or exceeds the permitted use, you will need to obtain permission directly from the copyright holder. To view a copy of this licence, visit http://creativecommons.org/licenses/by/4.0/. The Creative Commons Public Domain Dedication waiver (http://creativeco mmons.org/publicdomain/zero/1.0/) applies to the data made available in this article, unless otherwise stated in a credit line to the data. 
Table 1 Classification of muscle injuries in sports (according to $[4,5])$

A: Indirect muscle injuries
Type 1 and 2: functional/ultrastructural muscle disorder
Type 1: overexertion-related muscle disorder
Type 1a: fatigue-induced muscle disorders/muscular tightening/
hypertonicity
Type 1b: delayed-onset muscle soreness
Type 2: neuromuscular muscle disorder
Type 2a: spine-related neuromuscular disorders
Type 2b: muscle-related neuromuscular disorders/muscle strain
injury
Type 3 and 4: structural muscle injury
Type 3: partial muscle tears
Type 3a: minor partial muscle tear ( $<5$ mm; intrafascicle/bundle-tear)
Type 3b: moderate partial muscle tear ( $>5$ mm; interfascicle/bundle-
tear
Type 4: subtotal/complete muscle tear or tendinous avulsion
B: Direct muscle injuries
Contusion
Laceration

structural muscle injuries (smaller partial muscle tears) has largely been neglected in the academic literature during the last few decades, although these injuries can also cause considerable lay-off times of two weeks or longer $[2,8]$. In the guidelines for muscle injuries outlined by the Italian Society of Muscles, Ligaments and Tendons, Maffulli et al. [8] recommended a multimodal therapy approach comprising RICE (rest, ice, compression, elevation), optimized loading, manual therapy, functional compression bandages, low-level laser therapy, pulsed ultrasound therapy, electroanalgesia, training and functional rehabilitation, without reference to specific evidence in the literature or modifying the treatment plan to cater for different types of injury severity.

During the past few years, studies using animal subjects with acute muscle injuries and in vitro studies have shown that radial and focused extracorporeal shock wave therapy (rESWT, fESWT) may be of benefit in treating acute muscle injuries $[9,10]$. This form of treatment has already become well-established in successfully managing other pathologies of the musculoskeletal system, such as in the treatment of tendinopathies and fracture malunions [11, 12]. Radial and focused extracorporeal shock waves (rESWs, fESWs) are single acoustic impulses which have an initial high positive peak pressure between 10 and 100 megapascals that is reached in less than one microsecond, followed by a low tensile amplitude of a few $\mu$ s duration that can generate cavitation, and a short life cycle of approximately $10-20 \mu$ s $[13,14]$. Due to these characteristics rESWs and fESWs fundamentally differ from therapeutic ultrasound. Focused ESWs differ from
rESWs in terms of how the shock waves are generated. Focused ESWs also differ in terms of their physical characteristics and with regards to the penetration depth of the shock waves into the tissue [11, 14-16]. Studies on $\mathrm{rESWT} / \mathrm{fESWT}$ for the treatment of acute muscle injuries in elite football players (or other sportspeople) have not yet been published.

Based on anecdotal evidence we introduced rESWT (and to a much lesser extent, also fESWT) into our multimodal therapy approach for treating acute muscle injuries amongst elite football players (first/second German Bundesliga). Other components of this multimodal therapy approach included cryotherapy, compression, manual therapy, resistance/weight-training and a progressive physiotherapy exercise programme (in line with Maffulli et al. [8]).

In this study, we report our experience from the first entire season during which rESWT was applied. Our retrospective analysis was performed under the hypothesis that by integrating rESWT into our multimodal therapy approach, lay-off times may be shortened and a reduction in re-injury rates following acute functional/ultrastructural and structural muscle injuries amongst elite football players may be achieved.

\section{Methods \\ Participants}

We performed a retrospective analysis of treatments and time courses of all muscle injuries suffered by the players of an elite football team during one of the previous seasons (first/second German Bundesliga). This study was approved by the football club whose players were included in the study, and the local ethics board of Friedrich-Alexander University Erlangen-Nuremberg (Erlangen, Germany) (registration number 387_17 Bc). Written informed consent was obtained from all players for using their data in this study. CS did not visit the football club during diagnosis and treatment sessions and only had access to fully anonymous data.

All players were male and aged between 18 and 35 years old. All other details (including the dates of injury, side of injury, the player's position, whether an injured player was left-footed or right-footed, and whether an injury involved a player's support leg or kicking leg) are subject to confidentiality in order to protect the identity of each player within this study.

\section{Diagnosis and definition of acute muscle injuries}

The diagnosis of the acute muscle injuries within this study followed international guidelines and widely accepted clinical practice [8]. In summary, the diagnosis of functional/ultrastructural injuries (Type 1a and Type $2 \mathrm{~b}$ injuries, respectively) was based on clinical 
examination. Specifically, functional tests such as hopping on one leg were used to provoke pain as well as to test the player's readiness to commence (running) training; muscle length testing (stretch tests) and comparison with the non-injured limb, manual muscle strength testing and comparison with the non-injured limb, palpation of the injury site and comparison with the non-injured limb and a comprehensive subjective examination was undertaken. Pain was recorded using the Visual Analogue Scale (VAS) during the clinical examination. The subjective examination included noting the mechanism of injury and the exact injury location as well as noting the nature or quality of the pain being experienced (tension, tightness, sharp pain, burning, etc.). The diagnosis of Type $2 b, 3 a$ and $3 b$ injuries, respectively, was also performed by using the previously described clinical examination methods. In addition, ultrasound scans and magnetic resonance imaging (MRI) was used to differentiate between injury severity amongst these groups of injuries. Contusions were diagnosed based on the patient's history, clinical examination and ultrasound examination.

\section{Multimodal therapy approach for treating acute muscle injuries}

All acute muscle injuries were treated with a customized multimodal therapy approach comprising cryotherapy, compression, manual therapy, resistance/weight-training, a progressive physiotherapy exercise programme and ESWT (an example is provided in Table 2).

In 19 out of 20 cases rESWT was performed, using a Swiss DolorClast device (Electro Medical Systems, Nyon, Switzerland) equipped with an EvoBlue handpiece and 36-mm applicator. Radial ESWs were applied at $20 \mathrm{Hertz}(\mathrm{Hz})$. In the majority of cases rESWT was performed on a daily basis. The energy density of the rESWs was individually adjusted-so that the player reported some discomfort but did not experience pain during treatment, resulting in an air pressure of between 1.0 and 3.4 bar. A single treatment session consisted of between 6.000 and 12.000 rESWs being applied. Individual rESWT protocols are displayed in Fig. 1. The decision to incorporate rESWT protocols into our treatment plan for muscle injuries was based on our promising earlier clinical experience and observations as well as positive subjective player information

Table 2 Treatment protocol of a player who was diagnosed with a partial muscle tear (type $3 a$ ) of the soleus muscle (c.f. Figs. 1Q, $2 Q$ and 3)

\begin{tabular}{|c|c|c|c|}
\hline Day & Physiotherapy & Rehabilitation & rESWT \\
\hline 1 & Cryo, Comp & & \\
\hline 2 & Cryo, Comp & & \\
\hline 3 & Cryo, Comp & & \\
\hline 4 & Cryo, Comp, MT & Swimming & $7000 / 2.5$ \\
\hline 5 & Cryo, Comp, MT & Swimming & $7000 / 2.5$ \\
\hline 6 & Cryo, Comp, MT & Swimming, R/W/P triceps surae (TheraBand) & \\
\hline 7 & Cryo, Comp, MT & & $7000 / 2.5$ \\
\hline 8 & MT & Bicycling, R/W/P triceps surae (TheraBand) & $7000 / 3.0$ \\
\hline 9 & MT & Bicycling, R/W/P triceps surae (TheraBand) & \\
\hline 10 & MT & Bicycling, R/W/P triceps surae (training device) & \\
\hline 11 & MT & Bicycling & \\
\hline 12 & MT & Bicycling, R/W/P triceps surae (training device) & $7000 / 3.0$ \\
\hline 13 & MT & Running (10 1 min running followed by 1 min walking) & $7000 / 3.0$ \\
\hline 14 & & Running (10× 2 min running followed by 1 min walking) & \\
\hline 15 & MT & R/W/P triceps surae (training device) & $7000 / 3.2$ \\
\hline 16 & MT & Running $(2 \times 10 \mathrm{~min})$ & $7000 / 3.2$ \\
\hline 17 & MT & R/W/P triceps surae (training device) & \\
\hline 18 & MT & & $7000 / 3.2$ \\
\hline 19 & MT & R/W/P triceps surae (training device), running (20 min) & $7000 / 3.0$ \\
\hline 20 & MT & Running (30 min) & $7000 / 3.4$ \\
\hline 21 & & $\begin{array}{l}\text { Running ( } 40 \mathrm{~min}) \text { incl. } 4 \times 3 \mathrm{~min} \text { intervals at } 80 \% \text { of maximum heart-rate/ } \\
\text { lactate threshold }\end{array}$ & \\
\hline
\end{tabular}

rESWT, radial extracorporeal shock wave therapy (Swiss DolorClast (Electro Medical Systems, Nyon, Switzerland) with EvoBlue handpiece and 36-mm applicator): number of radial extracorporeal shock waves per session/air pressure (bar); Cryo, cryotherapy; Comp, compression; MT, manual therapy; R/W/P, resistance/weighttraining/progressive physiotherapy exercise programme 


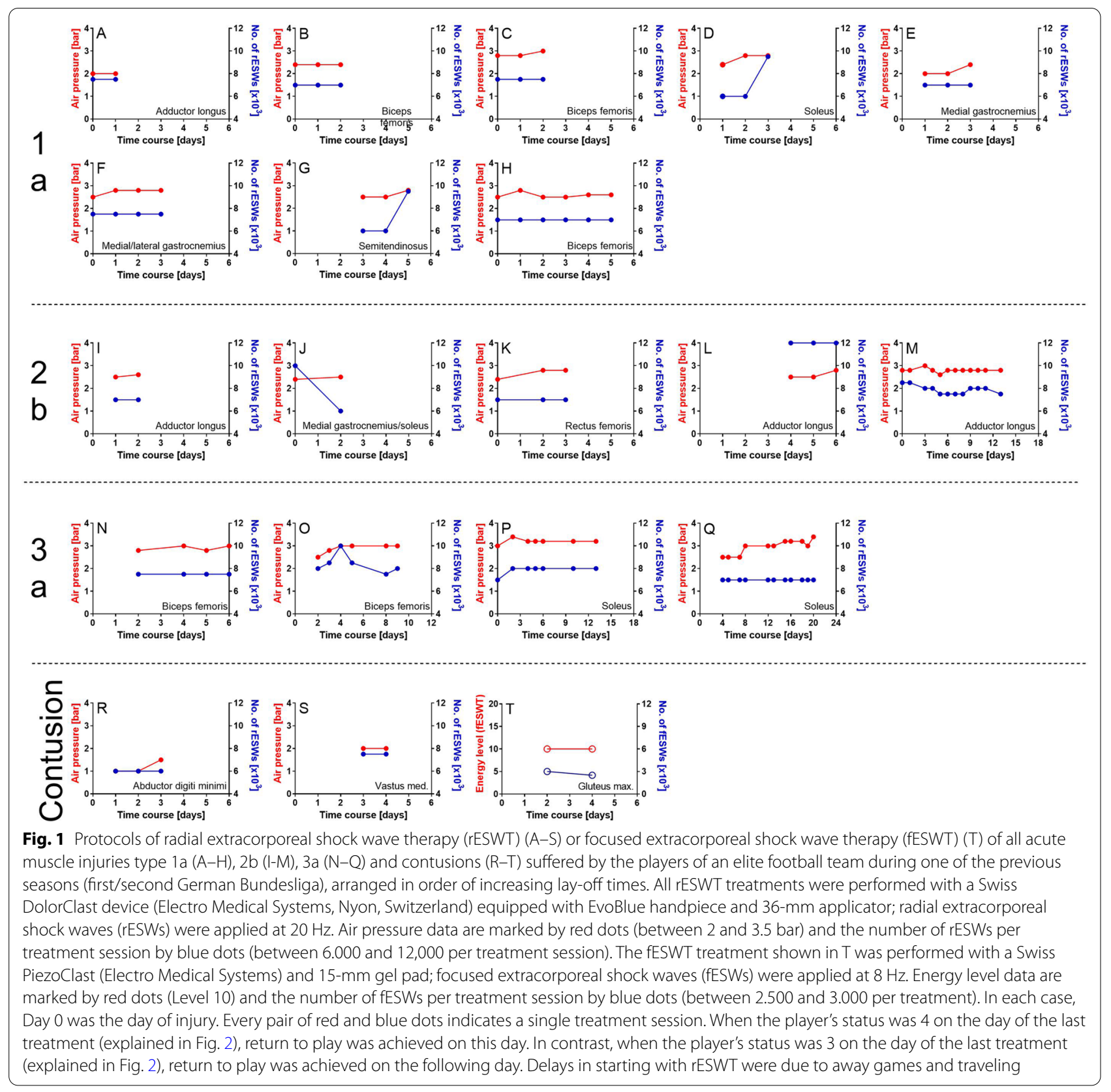

and feedback indicating that faster recovery times may be achievable in comparison to treatments of muscle injuries without the use of rESWT.

One case (contusion of the gluteus maximus muscle) was treated with fESWT, using a Swiss PiezoClast device (Electro Medical Systems) and 15-mm gel pad. Focused ESWs were applied at $8 \mathrm{~Hz}$; the positive energy density of the fESWs was 0.13 millijoule per square millimeter (energy level 10). A single treatment session consisted of between 2.500 and $3.000 \mathrm{fESWs}$ being applied. The corresponding fESWT protocol is shown in Fig. 1Q.
In the vast majority of cases rESWT/fESWT was commenced on the day of the injury $(9 / 20=45 \%)$, the day after the injury $(4 / 20=25 \%)$ or two days after the injury $(3 / 20=15 \%)$. Delays in starting with $\mathrm{rESWT} / \mathrm{fESWT}$ were due to away games or traveling and as a result not having immediate access to $\mathrm{rESWT} / \mathrm{fESWT}$.

There were no contraindications to rESWT/fESWT amongst the group of players that were treated. Contraindications to rESWT include as follows: local steroid injections during the last six weeks before rESWT/ fESWT, infection or tumor at the site of $\mathrm{rESWT} / \mathrm{fESWT}$ 
application, serious blood dyscrasia, blood-clotting disorders (including local thrombosis) and treatment with oral anticoagulants.

\section{Return to sport}

"Return-to-sport" status was defined as being once the player was able to fully participate in regular team training including contact training and was fully available for selection for matches in the first/second Bundesliga.

\section{Results}

A total of 20 acute muscle injuries occurred during the investigated season and were treated with the aforementioned approach, of which eight (40\%) were diagnosed as Type 1a injuries, five (25\%) as Type $2 \mathrm{~b}$ injuries, four (20\%) as Type 3a injuries and three (15\%) as contusions. Accordingly, $13 / 17=76 \%$ of the injuries that were not contusions were functional/ultrastructural injuries (Types $1 \mathrm{a}$ and $2 \mathrm{~b}$ ), and $4 / 17=24 \%$ were structural injuries (Type 3a). There were no Type 3b or Type 4 injuries suffered during the investigated season.

Amongst the 17 injuries that were not classified as contusions, one $(6 \%)$ occurred in the muscles in the anterior compartment of the thigh, four (24\%) in the muscles in the medial compartment of the thigh, six (35\%) in the posterior compartment of the thigh, and six $(35 \%)$ in the posterior compartment of the leg.

Injuries occurred during the entire season. There was no difference in the number of injuries suffered at the beginning/first half of the season compared to the injuries suffered in the second half of the season after the winter break. All of the structural injuries (Type 3a) occurred several months after the start of the season. The re-injury rate (defined as being an injury involving the same muscle and having the same severity as the initial injury occurring within two months after a player's return-to-sport following the initial injury [2]) was $1 / 8=12.5 \%$ for Type $1 \mathrm{a}$ injuries, and was zero in the case of Type 2b, 3a injuries and contusions, respectively. None of the Type $2 b$ injuries that were diagnosed occurred following a previously suffered Type 1a injury during the investigated season, and none of the Type 3a injuries occurred following a previously suffered Type $1 \mathrm{a}$ or $2 \mathrm{~b}$ injury during the investigated season.

Return-to-play was achieved after 3/3.3/2-6 days (median/mean/range), respectively, following Type 1a injuries, after 4/6.2/3-14 days, respectively, following Type $2 \mathrm{~b}$ injuries, after $12 / 13 / 7-22$ days, respectively, following Type $3 a$ injuries and after $4 / 4 / 4$ days, respectively, following contusions (Fig. 2).

The MRI pictures of the lower leg of a player who was diagnosed with a minor partial muscle tear (Type 3a) of the soleus muscle are shown in Fig. 3. In this case,
rESWT was performed on days 4, 5, 7, 8, 12, 13, 15, 16 and 18-20, respectively, following injury (Figs. 1Q and $2 \mathrm{Q})$. The detailed treatment protocol of this case is summarized in Table 2. Return-to-play was achieved on day 22 post-injury.

No adverse reactions to treatment were observed, except occasional, temporary reddening of the skin at the treatment site that disappeared within $24 \mathrm{~h}$ following rESWT.

All of the players who were treated for muscle injuries complied fully with the treatment protocol incorporating rESWT (or fESWT in one case). No player declined being treated with rESWT (or fESWT in one case) neither during the initial treatment nor during the follow-up treatments. Hence, by comparing the use of our treatment protocol for muscle injuries with other treatment protocols and the differences in lay-off times, respectively, one can conclude that non-compliance did not affect the treatment effect of our retrospective analysis.

\section{Discussion}

This is the first report concerning the course of recovery times following acute muscle injuries suffered by the players of an elite football team (competing in the first/ second German Bundesliga) during an entire season that incorporated $\mathrm{rESWT} / \mathrm{fESWT}$ into the treatment protocols of these injuries. A number of relevant conclusions can be drawn from these results (with reference to $\mathrm{rESWT}$ because fESWT was only used in one single case).

\section{Safety of integrating rESWT into a multimodal therapy approach for treating acute muscle injuries}

Integrating rESWT into a multimodal therapy approach for treating acute muscle injuries as outlined in this study is safe. There were no adverse reactions or complications to treatment observed apart from occasional, temporary reddening of the skin at the treatment site that disappeared within $24 \mathrm{~h}$ following rESWT. Moreover, there were no observed cases of local hematoma following the application of rESWs - as has been previously reported in the literature following the application of rESWs for the treatment of the gluteus maximus muscle with a rESWT device that differed from the one that was used in this study [17]. In addition, none of the players that were treated suffered from myositis ossificans (MO)-a proliferative mesenchymal response following soft tissue trauma that causes localized ossification [18]. This is important to note because, at first glance, exposure of injured muscles to rESWs could be considered inappropriate physiotherapy [19]. Walczak et al. [18] hypothesized (with reference to a study by Medici et al. [20]) that the development of MO may depend on a process 

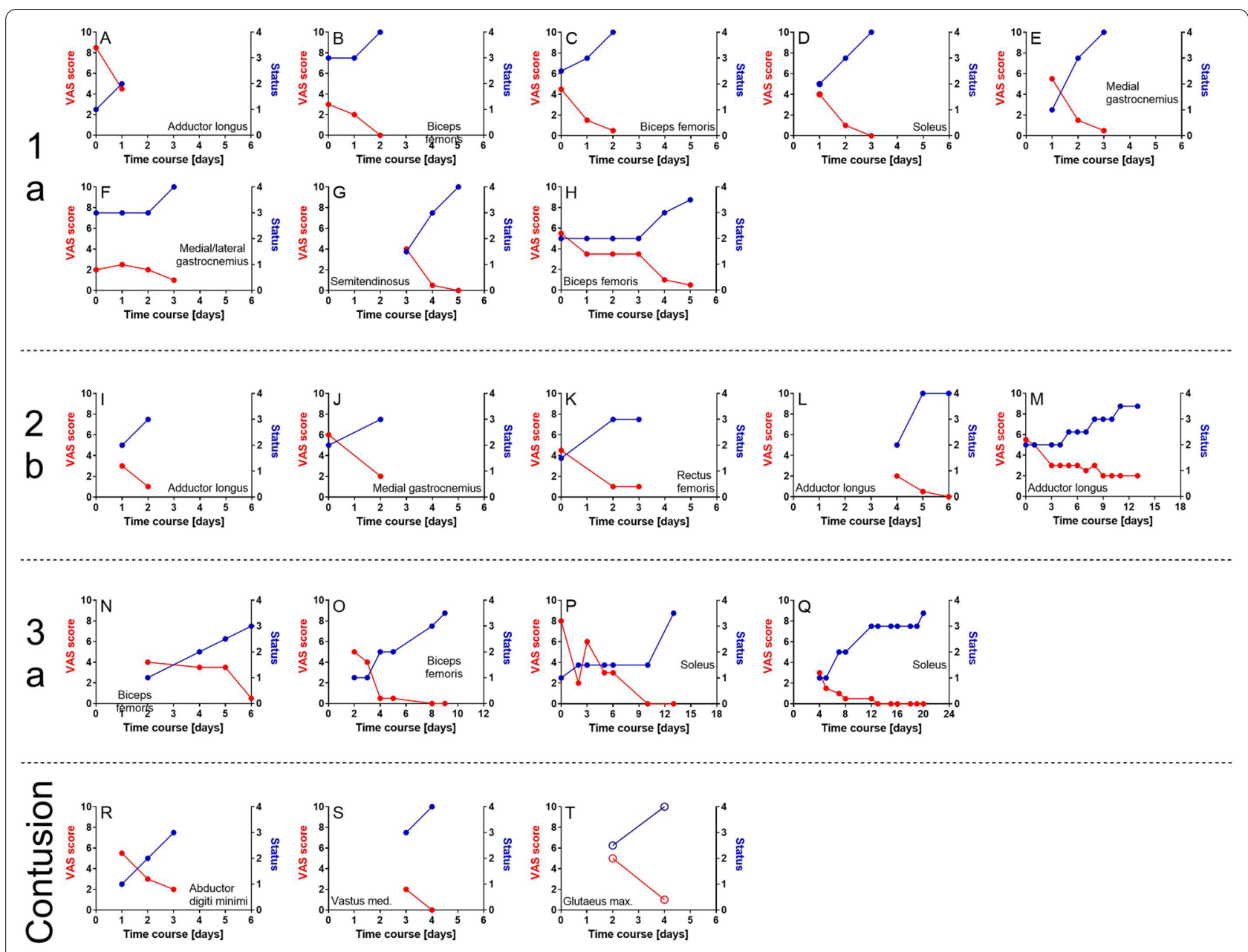

Fig. 2 Time course of treatments of acute muscle injuries Type $1 a(A-H), 2 b(I-M), 3 a(N-Q)$ and contusions (R-T) suffered by the players of an elite football team during one of the previous seasons (first/second German Bundesliga), arranged in order of increasing lay-off times. VAS scores are marked by red dots $(0=$ no pain; $10=$ maximal pain $)$ and the player's status by blue dots $(1=$ injured; $2=$ rehabilitation; $3=$ training; $4=$ fully fit/return to play). Diagrams A-S represent treatments with radial extracorporeal shock wave therapy (rESWT), whereas Diagram T represents treatments with focused extracorporeal shock wave therapy (fESWT). In every case Day 0 was the day of injury, and a pair of red and blue dots indicates a single treatment session. When the player's status was 4 on the day of the last treatment, return to play was achieved on this day. In contrast, when the player's status was 3 on the day of the last treatment, return to play was achieved on the following day. Delays in commencing rESWT/fESWT were due to away games and traveling

called endothelial-mesenchymal transition. According to this hypothesis, skeletal muscle injury may induce a local inflammatory cascade, which leads to the release of bone morphogenetic protein (BMP)-2, BMP-4 and transforming growth factor (TGF). These cytokines may act on vascular endothelial cells and induce endothelialmesenchymal transition. As a result, endothelial-derived mesenchymal stem cells may differentiate into osteoblasts and chondrocytes when exposed to an inflammatory-rich environment [18]. Accordingly, it is important to note that exposure of tissue to rESWs/fESWs (or, more specifically, exposure to fESWs; because related studies on rESWs have not yet been published) can increase local concentrations of BMP-2, BMP-4 and TGF- 31 [21, 22]. If this is the case it would clearly be an argument against using $\mathrm{rESWT} / \mathrm{fESWT}$ in the treatment of acute muscle injuries. However, it is important to keep in mind that the aforementioned hypothesis of endothelial-mesenchymal transition in the pathophysiology of MO is based on observations of a rare disease called fibrodysplasia ossificans progressiva [20] rather than on traumatic MO. A number of studies on human heterotopic ossification and related mouse models have demonstrated that bone marrow-derived osteoblast progenitor cells in circulating blood may contribute to the formation of heterotopic bone [23-25]. Moreover, a recent study involving 

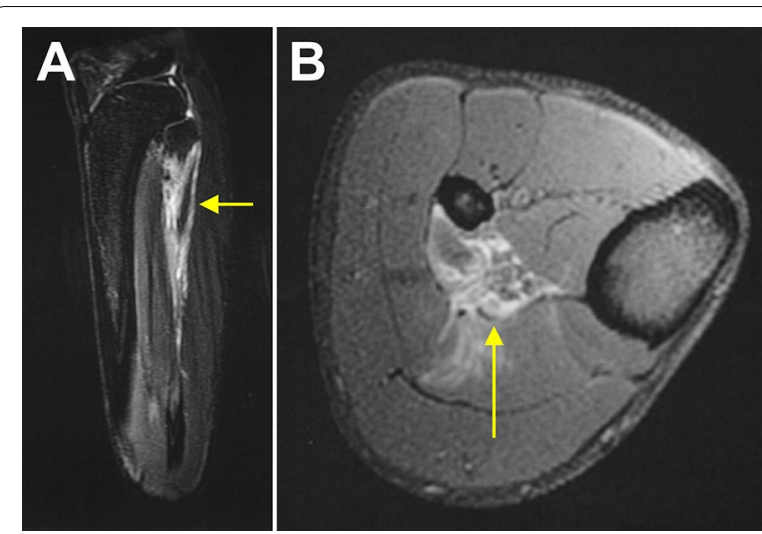

Fig. 3 MRI images of the lower leg of an elite football player who was diagnosed with a minor partial muscle tear (Type 3a) of the right soleus muscle (yellow arrows). The time course of the rehabilitation of this player is shown in Fig. 1Q; the detailed treatment protocol is summarized in Table 2. Return to play was achieved on Day 22 post-injury

a parabiosis model (i.e., two mice with shared circulation, in which the cells of one mouse can be labeled and detected in the other mouse when released into the bloodstream) has demonstrated that these bone marrowderived osteoblast progenitor cells do not contribute to early stage development of heterotopic ossification [26]. The authors of this study argued that a timely intervention that influences the molecular and cellular processes that contribute to the recruitment and/or differentiation of the circulating cell populations that participate in heterotopic ossification may block the initiation of the latter and ultimately prevent the transition to definitive bone [26]. We believe that this may be achieved by the very early treatment of acute muscle injuries with rESWT, as performed in this study. Further studies on animal models are required to test this hypothesis. It is worth noting that in this study commencing rESWT immediately following Type $1 \mathrm{a}$ and $2 \mathrm{~b}$ injuries did not lead to inferior results when compared with starting treatment with rESWT on days 1, 3 or 4 post-injury (delay in commencing treatment was due to some injuries being suffered during away games that required traveling after the games and as a result there being no immediate access to rESWT/fESWT).

\section{Shortening of lay-off times by integrating rESWT into a multimodal therapy approach for treating acute muscle injuries}

Integrating rESWT into a multimodal therapy approach for the treatment of acute muscle injuries as performed in this study may shorten lay-off times for elite football players (as well as for other sportspeople) compared to other therapy approaches. This conclusion is based on a comparison of the results of this study with data that were obtained prospectively from 31 European elite male football teams during the 2011/2012 season (Table 3) [2]

A direct comparison with the data reported by Ekstrand et al. [2] shows that the median/mean lay-off times in this study were shortened by $54 \% / 58 \%$, respectively, in the case of Type 1a injuries, by $50 \% / 55 \%$, respectively, in the case of Type $2 \mathrm{~b}$ injuries, and by $8 \% / 21 \%$, respectively, in the case of Type 3a injuries. Arguably, the Type 3a structural injury shown in Fig. 3 could also be considered to be a more severe Type 3b injury rather than a Type 3a injury which would mean that the median/mean injury lay-off times in this study for Type 3a injuries would then be further shortened by $23 \% / 37 \%$, respectively, compared to the data reported by Ekstrand et al. [2]. Furthermore, in recently published guidelines for muscle injuries, Maffulli et al. [8] consider normal lay-off times to be $5-15$ days for functional/ultrastructural injuries, 15-18 days for Type 3a injuries and 25-35 days for Type 3b injuries. The layoff times reported in this study were substantially shorter than the aforementioned typical lay-off times noted by Maffulli et al. [8]. At this stage, it is difficult to ascertain to which extent the application of rESWs actually

Table 3 Comparison of lay-off times after acute muscle injuries in elite football players reported in this study as well as by Ekstrand et al. [2]

\begin{tabular}{|c|c|c|c|c|c|c|c|c|c|c|}
\hline & Type & & & Type & & & Type 3a & & & \\
\hline & R-TS & R-E & PD & R-TS & R-E & PD & R-TS & R-E (3a) & PD & $R-E(3 b)$ \\
\hline$n$ & 8 & 82 & & 5 & 22 & & $4(3)$ & 192 & & 66 \\
\hline Median & 3 & 6.5 & $-54 \%$ & 4 & 8 & $-50 \%$ & $12(10)$ & 13 & $-8 \%(-23 \%)$ & 32 \\
\hline Mean & 3.3 & 7.9 & $-58 \%$ & 6.2 & 13.8 & $-55 \%$ & $13(10.3)$ & 16.4 & $-21 \%(-37 \%)$ & 37.9 \\
\hline SD & 1.5 & 7.4 & & 4.7 & 20.6 & & $6.1(3.5)$ & 17.1 & & 24.0 \\
\hline Min & 2 & 1 & & 3 & 2 & & 7 & 2 & & 8 \\
\hline Max & 6 & 58 & & 14 & 100 & & 14 & 132 & & 156 \\
\hline
\end{tabular}

R-TS, results of this study; R-E, results reported by Ekstrand et al. [2]; PD, percentage difference 
contributed to the shorter lay-off times in this study compared to the data reported by Ekstrand et al. [2]. This is partly due to the fact that no treatment protocols were outlined in the latter study. Nevertheless, several factors strengthen the argumentation that the application of rESWs may be helpful as an adjunct form of therapy for reducing lay-off times following muscle injuries. Specifically: (1) the study by Ekstrand et al. [2] was based on data that was obtained from European male elite football teams and, therefore, from a population that is very similar to the population investigated in this study; (2) the key molecular and cellular mechanisms of action that rESWs/ fESWs have on muscles (outlined below) were not yet known in 2011/2012; (3) Maffulli et al. [8] did not mention $\mathrm{rESWT} / \mathrm{fESWT}$ in their guidelines for muscle injuries; and (4) our multimodal therapy approach (excluding $\mathrm{rESWT} / \mathrm{fESWT}$ ) is in line with the guidelines published by Maffulli et al. [8]. With this in mind, it is reasonable to assume that the therapy approaches used in 2011/2012 by the teams who were investigated by Ekstrand et al. [2] were broadly comparable to the multimodal therapy approach (excluding rESWT/fESWT) used in this study. Hence the application of rESWs may have substantially contributed to the shorter lay-off times found in this study compared to the data reported by Ekstrand et al. [2]. Further studies are required to test this hypothesis.

\section{Reduction in total re-injury rates by integrating rESWT into a multimodal therapy approach for treating acute muscle injuries}

the total re-injury rates of $1 / 13(8 \%)$ observed amongst functional/ultrastructural muscle injuries and $0 / 4(0 \%)$ observed amongst the structural muscle injuries in this study were lower than corresponding data reported by Ekstrand et al. [2] (12\% was observed amongst functional/ultrastructural injuries and 13\% observed amongst structural injuries, respectively). Based on these results we argue that the integration of rESWT into a multimodal therapy approach for the treatment of acute muscle injuries as outlined in this study may not only help to reduce lay-off times but may also help in the prevention of muscle re-injury amongst athletes. However, due to the low number of structural muscle injuries included in this study, further studies are required to test this hypothesis amongst a larger sample group.

In addition, no Type $2 b$ injuries occurred in this study following a Type 1a injury and no structural injury occurred following a functional/ultrastructural injury. In contrast, Ekstrand et al. [2] reported in their study, that $5 \%$ of the initial functional/ultrastructural injuries developed into secondary structural injuries within two months of the primary injury. However, they did not report how many Type $2 \mathrm{~b}$ injuries occurred following an earlier Type 1a injury during the investigated season, nor did they comment on how many structural injuries occurred following previously suffered functional/ultrastructural injuries during the entire investigated season (i.e., not only including the initial two months, or "reinjury" period following the primary injury). It is important to note that in this study, the number of functional/ ultrastructural compared to structural acute muscle injuries was 13 as opposed to four. Hence there were considerably more functional/ultrastructural injuries included in this study, but this number was 130 as opposed to 263 (i.e., more structural injuries) in the study by Ekstrand et al. [2]. Of course, many other factors may have contributed to this finding-such as differences in the management of training load upon returning to team training, and other prevention strategies used such as optimizing nutrition and using screening protocols for detecting early warning signs that may allow sport medical staff to help "forecast" an impending muscle injury [27-29]. In a recent study regular eccentric lengthening of the muscles in the lower limbs as performed in the practice of Salah (a religious prayer amongst Muslims) was found to reduce the number of structural muscle injuries amongst professional Russian football players when compared with a control group [30]. Exercises involving the eccentric lengthening of muscles were also used as a component of the physiotherapy exercises integrated into the multimodal therapy approach used in this study. Furthermore, decreasing delayed onset muscle soreness by whole body vibration could also contribute to reduced muscle injury risk in elite football players, as recently shown in elite hockey players [31]. However, one cannot rule out that the integration of rESWT into a multimodal therapy approach for the treatment of acute muscle injuries as performed in this study may also contribute to the prevention of structural muscle injuries in athletes.

\section{Limitations}

This study is an audit of retrospectively collected data, and therefore has a number of inherent limitations. Firstly, there was no control group. The reason for this was that the evidence that was available at the time when we decided to integrate rESWT/fESWT into our therapy approach was not sufficient enough to gain approval from the club for a possible prospective study with a control group. Paradoxically, the promising results of this study (in particular the comparison to the data reported by Ekstrand et al. [2]) may now also cause difficulties in gaining approval from the same or another club for a corresponding prospective study with a control group, as the clubs' interests obviously lie in reducing lay-off times following injuries to a minimum. Moreover, gaining approval for a corresponding randomized controlled trial 
(RCT) from the players themselves may also prove to be more difficult following the initial good results obtained from using rESWT/fESWT in this study. Perhaps it is more realistic to expect that future RCTs be performed on recreational athletes, as in the case of one current study that relates to the treatment of acute Type $3 \mathrm{~b}$ hamstring muscle injuries [16].

Secondly, with the exception of one case (contusion) only rESWT was investigated in this study. One may argue that fESWT may also be effective in the management of acute muscle injuries amongst athletes, but obviously there are not enough data to support this hypothesis. Our decision to focus on rESWT rather than on fESWT was based on the fact that the data relating to treatment of other pathologies of the musculoskeletal system (tendinopathies and fracture malunions of superficial bones) do not support the hypothesis that fESWT is superior to rESWT [11, 12]. In addition, the use of fESWT is restricted to physicians in many countries (as is the case in Germany where physiotherapists and chiropractors who have been trained in Germany are not legally entitled to use fESWT). Moreover, the International Society for Medical Shockwave Treatment (ISMST) has recommended that only a qualified physician (certified by National or International Societies) may use fESWT in their latest Consensus Statement regarding ESWT indications and contraindications [32]. However, in many elite football clubs (and training centers involving other sporting codes) a medical doctor may not be available to perform treatment on a daily basis. This is also the reason why a RCT on acute Type $3 \mathrm{~b}$ hamstring muscle injuries currently being undertaken is based on rESWT rather than on fESWT [16].

Thirdly, only one rESWT protocol was applied in this study, and this protocol differed considerably from other published rESWT protocols used in the treatments of tendinopathies [11]. Specifically, there were differences regarding the timing with regards to commencing rESWT (immediately after the injury in this study compared with starting treatment six or more months after initial diagnosis and following unsuccessful treatment involving other conservative modalities [33-35]), differences in the time interval between treatment sessions (in most cases daily treatment sessions were used in this study as compared to one treatment session per week used in other studies [11]) and differences in the number of rESWs applied per treatment session (6.000-12.000 in this study as opposed to treatments averaging 2.000) [11]. As already mentioned above, our motivation to use this particular rESWT protocol was based on our (and the players') clinical experience and observations that players achieved faster recovery times than in our earlier treatments of muscle injuries that did not include using rESWT (which has been confirmed by comparing the data set in this study with the data by Ekstrand et al. [2]). Nevertheless, we cannot rule out the possibility that a time interval of two days between treatment sessions, the application of fewer than $6.000 \mathrm{rESWs}$ per treatment session and/or application of rESWs with lower energy densities than those applied in this study may lead to the same results (reduced lay-off times, reduced reinjury rates, etc.) in the treatment of acute muscle injuries amongst elite football players as reported in this study. This may be addressed in follow-up studies.

Finally, the precise molecular and cellular mechanisms that may have contributed to the outcomes of this study as a result of incorporating rESWT into our treatment protocol remain unknown (there were no muscle biopsies or blood samples taken in this study). In addition, there have been no studies published using animal models with similar muscle injuries as those that were investigated in this study in which rESWT was applied in conjunction with other treatment modalities that would mimic our multimodal therapy approach. The molecular and cellular mechanisms of rESWT/fESWT include: (1) the depletion of presynaptic substance $\mathrm{P}$ from $\mathrm{C}$-fibers [36] leading to a reduction in the sensation of pain and blockage of neurogenic inflammation [37]; (2) muscular relaxation possibly caused by the mechanical separation of actin and myosin filaments and/or transient dysfunction of nerve conduction at neuromuscular junctions [38, 39]; (3) stimulation of tissue remodeling by promoting inflammatory and catabolic processes that are associated with the removal of damaged matrix constituents [40]; (4) enhanced proliferation and differentiation rates and modulation of gene expression of muscle satellite cells $[9,10]$; (5) stimulation of fibroblast proliferation [14]; (6) improved fascial gliding within the surrounding tissues due to increased lubricin expression [41]; and (7) functional angiogenesis/ improved blood circulation [42, 43]. Other studies that have used therapies in order to improve musculoskeletal healing at a cellular level have largely included injecting platelet-rich plasma at the injury site; however, the evidence to support this form of intervention is poor [44, 45]. In contrast, the aforementioned positive effects of applying rESWT to injured muscle as carried out in this study may have been an important factor in supporting the musculoskeletal healing process. Nevertheless, many of these mechanisms may also have a positive carryover on the other treatment modalities used in our multimodal therapy approach. For example, pain reduction may allow manual therapy to be carried out more effectively, and improved fascial movement may contribute to better performance during training. These assumptions need to be supported by further studies. 


\section{Conclusions}

This study suggests that integrating rESWT into a multimodal therapy approach for the treatment of acute type $1 \mathrm{a}, 2 \mathrm{~b}, 3 \mathrm{a}$ muscle injuries and contusions is safe and effective, and leads to shortened lay-off times and reduced re-injury rates amongst elite football players, without causing any adverse effects. Clinicians should consider rESWT in the management of acute type $1 \mathrm{a}, 2 \mathrm{~b} 3 \mathrm{a}$ muscle injuries and contusions in athletes and sportspeople.

\section{Abbreviations}

BMP: Bone morphogenetic protein; Comp: Compression; Cryo: Cryotherapy; fESWs: Focused extracorporeal shock waves; fESWT: Focused extracorporeal shock wave therapy; Hz: Hertz; MO: Myositis ossificans; MRI: Magnetic resonance imaging; MT: Manual therapy; PD: Percentage difference; RCT: Randomized controlled trial; R-E: Results reported by Ekstrand et al. [2]; rESWs: Radial extracorporeal shock waves; rESWT: Radial extracorporeal shock wave therapy; R-TS: Results of this study; R/W/P: Resistance/weight-training/progressive physiotherapy exercise programme; TGF: Transforming growth factor.
\end{abstract}

\section{Acknowledgements}

We would like to give special thanks to the athletes whose data were analyzed in this study for their cooperation and support.

\begin{abstract}
Authors' contributions
JPMM performed all treatments and documented treatment outcome, reviewed and revised the manuscript. CS drafted the manuscript. $\mathrm{MH}$ and $\mathrm{MHB}$ reviewed and revised the manuscript. JPMM, MH, CS and MHB designed the study, analyzed and interpreted the data. All authors read and approved the final manuscript.
\end{abstract}

\section{Funding}

Not applicable.

\section{Availability of data and materials}

Due to confidentiality reasons, there are no data that can be shared.

\section{Declarations}

\section{Ethics approval and consent to participate}

This study was performed according to the Declaration of Helsinki. The study was approved by the local ethics board of Friedrich-Alexander University Erlangen-Nuremberg (Erlangen, Germany) (registration number 387_17 BC). All players whose data were analyzed in this study have explicitly granted permission, including publication of the data.

\section{Consent for publication}

All players whose individual healing process and course of treatment are shown in Figs. 1 and 2 as well as the player whose individual MRI scans are shown in Fig. 3 have explicitly granted permission to publish these data and images.

\section{Competing interests}

CS has received research funding from Electro Medical Systems (Nyon, Switzerland) (the inventor, manufacturer and distributor of the Swiss DolorClast rESWT device as well as the distributor of the Swiss PiezoClast fESWT device) for his preclinical research at LMU Munich (unrestricted grant) and consulted (until December 31, 2017) for Electro Medical Systems. Furthermore, Electro Medical Systems provided the rESWT and fESWT devices used in this study. However, Electro Medical Systems had no role in study design, data collection and analysis, interpretation of the data, and no role in the decision to publish and write this manuscript. No other potential competing interest relevant to this article were reported.

\section{Author details}

${ }^{1}$ Chair of Neuroanatomy, Institute of Anatomy, Faculty of Medicine, Extracorporeal Shock Wave Research Unit, LMU Munich, Munich, Germany. ${ }^{2}$ Task Force "Future of Professional Football", DFL Deutsche Fussball Liga, Frankfurt, Germany. ${ }^{3}$ Curathleticum Clinic, Nuremberg, Germany. ${ }^{4}$ Division of Trauma Surgery, Department of Surgery, Faculty of Medicine, University Hospital Erlangen, Friedrich-Alexander University Erlangen-Nuremberg, Erlangen, Germany.

Received: 16 September 2021 Accepted: 23 November 2021

Published online: 07 December 2021

\section{References}

1. Ekstrand J, Hägglund M, Waldén M. Epidemiology of muscle injuries in professional football (soccer). Am J Sports Med. 2011;39(6):1226-32. https://doi.org/10.1177/0363546510395879.

2. Ekstrand J, Askling C, Magnusson H, Mithoefer K. Return to play after thigh muscle injury in elite football players: implementation and validation of the Munich muscle injury classification. Br J Sports Med. 2013;47(12):769-74. https://doi.org/10.1136/bjsports-2012-092092.

3. Maffulli N, Del Buono A, Oliva F, Giai Via A, Frizziero A, Barazzuol M, Brancaccio P, Freschi M, Galletti S, Lisitano G, Melegati G, Nanni G, Pasta G, Ramponi C, Rizzo D, Testa V, Valent A. Muscle injuries: a brief guide to classification and management. Transl Med UniSa. 2014;12:14-8.

4. Müller-Wohlfahrt HW, Ueblacker P, Haensel L, Garrett JWE. Muscle injuries in sports. 1st ed. Stuttgart: Thieme; 2013. p. 432.

5. Sorg T, Best R. Muskelläsion: Exakte Diagnose erlaubt gute Einschätzung des Heilverlaufs [Muscle lesion: exact diagnosis allows good assessment of the healing process]. Orthopädie \& Rheuma. 2018;21:22-7. German.

6. Reurink G, Goudswaard GJ, Moen MH, Weir A, Verhaar JA, Bierma-Zeinstra $\mathrm{SM}$, et al. Platelet-rich plasma injections in acute muscle injury. New Engl J Med. 2014;370(26):2546-7. https://doi.org/10.1056/NEJMc1402340.

7. Bayer ML, Magnusson SP, Kjaer M; Tendon Research Group Bispebjerg. Early versus delayed rehabilitation after acute muscle injury. N Engl J Med. 2017;377(13):1300-1. DOl: https://doi.org/10.1056/NEJMc1708134

8. Maffulli N, Oliva F, Frizziero A, Nanni G, Barazzuol M, Via AG, et al. ISMuLT Guidelines for muscle injuries. Muscles Ligaments Tendons J. 2014:3(4):241-9.

9. Zissler A, Steinbacher P, Zimmermann R, Pittner S, Stoiber W, Bathke AC, et al. Extracorporeal shock wave therapy accelerates regeneration after acute skeletal muscle injury. Am J Sports Med. 2017;45(3):676-84. https:// doi.org/10.1177/0363546516668622.

10. Mattyasovszky SG, Langendorf EK, Ritz U, Schmitz C, Schmidtmann I, Nowak TE, et al. Exposure to radial extracorporeal shock waves modulates viability and gene expression of human skeletal muscle cells: a controlled in vitro study. J Orthop Surg Res. 2018;13(1):75. https://doi.org/10.1186/ s13018-018-0779-0.

11. Schmitz C, Császár NB, Milz S, Schieker M, Maffulli N, Rompe JD, et al. Efficacy and safety of extracorporeal shock wave therapy for orthopedic conditions: a systematic review on studies listed in the PEDro database. Br Med Bull. 2015;116(1):115-38. https://doi.org/10.1093/bmb/ldv047.

12. Kertzman P, Császár NBM, Furia JP, Schmitz C. Radial extracorporeal shock wave therapy is efficient and safe in the treatment of fracture nonunions of superficial bones: a retrospective case series. J Orthop Surg Res. 2017;12(1):164. https://doi.org/10.1186/s13018-017-0667-z.

13. Császár NB, Angstman NB, Milz S, Sprecher CM, Kobel P, Farhat M, et al. Radial shock wave devices generate cavitation. PLoS ONE. 2015;10(10): e0140541. https://doi.org/10.1371/journal.pone.0140541.

14. Hochstrasser T, Frank HG, Schmitz C. Dose-dependent and cell typespecific cell death and proliferation following in vitro exposure to radial extracorporeal shock waves. Sci Rep. 2016;6:30637. https://doi.org/10. 1038/srep30637.

15. Ogden JA, Toth-Kischkat A, Schultheiss R. Principles of shock wave therapy. Clin Orthop Relat Res. 2001;387:8-17. https://doi.org/10.1097/ 00003086-200106000-00003.

16. Crupnik J, Silveti S, Wajnstein N, Rolon A, Vollhardt A, Stiller P, et al. Is radial extracorporeal shock wave therapy combined with a specific rehabilitation program (rESWT + RP) more effective than sham-rESWT + RP for acute hamstring muscle complex injury type $3 b$ in athletes? Study protocol for a prospective, randomized, double-blind, sham-controlled 
single centre trial. J Orthop Surg Res. 2019;14(1):234. https://doi.org/10. 1186/s13018-019-1283-x.

17. Gleitz M, Hornig K. Triggerpunkte - Diagnose und Behandlungskonzepte unter besonderer Berücksichtigung extrakorporaler Stoßwellen [Trigger points - Diagnosis and treatment concepts with special reference to extracorporeal shockwaves]. Orthopäde. 2012;41(2):113-25. https://doi. org/10.1007/s00132-011-1860-0.

18. Walczak BE, Johnson CN, Howe BM. Myositis ossificans. J Am Acad Orthop Surg. 2015;23(10):612-22. https://doi.org/10.5435/JAAOS-D-14-00269.

19. King JB. Post-traumatic ectopic calcification in the muscles of athletes: a review. Br J Sports Med. 1998;32(4):287-90. https://doi.org/10.1136/bjsm. 32.4.287.

20. Medici D, Olsen BR. The role of endothelial-mesenchymal transition in heterotopic ossification. J Bone Miner Res. 2012;27(8):1619-22. https:// doi.org/10.1002/jbmr.1691.

21. Wang FS, Yang KD, Kuo YR, Wang CJ, Sheen-Chen SM, Huang HC, et al. Temporal and spatial expression of bone morphogenetic proteins in extracorporeal shock wave-promoted healing of segmental defect. Bone. 2003;32(4):387-96. https://doi.org/10.1016/s8756-3282(03)00029-2.

22. Wang CJ, Yang KD, Ko JY, Huang CC, Huang HY, Wang FS. The effects of shockwave on bone healing and systemic concentrations of nitric oxide (NO), TGF-beta1, VEGF and BMP-2 in long bone non-unions. Nitric Oxide. 2009;20(4):298-303. https://doi.org/10.1016/j.niox.2009.02.006.

23. Otsuru S, Tamai K, Yamazaki T, Yoshikawa H, Kaneda Y. Bone marrowderived osteoblast progenitor cells in circulating blood contribute to ectopic bone formation in mice. Biochem Biophys Res Commun. 2007;354(2):453-8. https://doi.org/10.1016/j.bbrc.2006.12.226.

24. Otsuru S, Tamai K, Yamazaki T, Yoshikawa H, Kaneda Y. Circulating bone marrow-derived osteoblast progenitor cells are recruited to the boneforming site by the CXCR4/stromal cell-derived factor-1 pathway. Stem Cells. 2008;26(1):223-34. https://doi.org/10.1634/stemcells.2007-0515.

25. Egan KP, Duque G, Keenan MA, Pignolo RJ. Circulating osteogenetic precursor cells in non-hereditary heterotopic ossification. Bone. 2018;109:61-4. https://doi.org/10.1016/j.bone.2017.12.028.

26. Loder SJ, Agarwal S, Chung MT, Cholok D, Hwang C, Visser N, et al. Characterizing the circulating cell populations in traumatic heterotopic ossification. Am J Pathol. 2018;188(11):2464-73. https://doi.org/10.1016/j. ajpath.2018.07.014.

27. Close GL, Sale C, Baar K, Bermon S. Nutrition for the prevention and treatment of injuries in track and field athletes. Int I Sport Nutr Exerc Metab. 2019;29(2):189-97. https://doi.org/10.1123/ijsnem.2018-0290.

28. Smyth EA, Newman P, Waddington G, Weissensteiner JR, Drew MK. Injury prevention strategies specific to pre-elite athletes competing in Olympic and professional sports-A systematic review. J Sci Med Sport. 2019;22(8):887-901. https://doi.org/10.1016/j.jsams.2019.03.002.

29. Jones CM, Griffiths PC, Mellalieu SD. Training load and fatigue marker associations with injury and illness: a systematic review of longitudinal studies. Sports Med. 2017;47(5):943-74. https://doi.org/10.1007/ s40279-016-0619-5.

30. Bezuglov E, Talibov O, Butovskiy M, Lyubushkina A, Khaitin V, Lazarev A, Achkasov E, Waśkiewicz Z, Rosemann T, Nikolaidis PT, Knechtle B, Maffulli $\mathrm{N}$. The prevalence of non-contact muscle injuries of the lower limb in professional soccer players who perform Salah regularly: a retrospective cohort study. J Orthop Surg Res. 2020;15(1):440. https://doi.org/10.1186/ s13018-020-01955-5.

31. Akehurst $\mathrm{H}$, Grice JE, Angioi M, Morrissey D, Migliorini F, Maffulli N. Whole-body vibration decreases delayed onset muscle soreness following eccentric exercise in elite hockey players: a randomised controlled trial. J Orthop Surg Res. 2021;16(1):589. https://doi.org/10.1186/ s13018-021-02760-4.

32. Eid J. Consensus Statement on ESWT Indications and Contraindications, published on 12 October 2016 (cited 16 September 2020). Available from: https://www.shockwavetherapy.org/fileadmin/user_upload/dokumente/ PDFs/Formulare/ISMST_consensus_statement_on_indications_and contraindications_20161012_final.pdf.

33. Gerdesmeyer L, Frey C, Vester J, Maier M, Weil L Jr, Weil L Sr, et al. Radial extracorporeal shock wave therapy is safe and effective in the treatment of chronic recalcitrant plantar fasciitis: results of a confirmatory randomized placebo-controlled multicenter study. Am J Sports Med. 2008;36(11):2100-9. https://doi.org/10.1177/0363546508324176.
34. Thomas JL, Christensen JC, Kravitz SR, Mendicino RW, Schuberth JM, Vanore JV, et al. The diagnosis and treatment of heel pain: a clinical practice guideline-revision 2010. J Foot Ankle Surg. 2010;49(3 Suppl):S1-19. https://doi.org/10.1053/j.jfas.2010.01.001.

35. Rompe JD, Furia J, Cacchio A, Schmitz C, Maffulli N. Radial shock wave treatment alone is less efficient than radial shock wave treatment combined with tissue-specific plantar fascia-stretching in patients with chronic plantar heel pain. Int J Surg. 2015;24(Pt B):135-42. https://doi.org/ 10.1016/j.ijsu.2015.04.082.

36. Maier M, Averbeck B, Milz S, Refior HJ, Schmitz C. Substance P and prostaglandin E2 release after shock wave application to the rabbit femur. Clin Orthop Relat Res. 2003;406:237-45. https://doi.org/10.1097/01.blo.00000 30173.56585.8f.

37. Snijdelaar DG, Dirksen R, Slappendel R, Crul BJP. Substance P. Eur J Pain. 2000;4(2):121-35. https://doi.org/10.1053/eujp.2000.0171.

38. Kenmoku T, Nemoto N, Iwakura N, Ochiai N, Uchida K, Saisu T, et al. Extracorporeal shock wave treatment can selectively destroy end plates in neuromuscular junctions. Muscle Nerve. 2018;57(3):466-72. https://doi. org/10.1002/mus.25754.

39. Angstman NB, Kiessling MC, Frank HG, Schmitz C. High interindividual variability in dose-dependent reduction in speed of movement after exposing C. elegans to shock waves. Front Behav Neurosci. 2015;9:12. https://doi.org/10.3389/fnbeh.2015.00012.

40. Waugh CM, Morrissey D, Jones E, Riley GP, Langberg H, Screen HR. In vivo biological response to extracorporeal shockwave therapy in human tendinopathy. Eur Cell Mater. 2015;29:268-80. https://doi.org/10.22203/ ecm.v029a20.

41. Zhang D, Kearney CJ, Cheriyan T, Schmid TM, Spector M. Extracorporeal shockwave-induced expression of lubricin in tendons and septa. Cell Tissue Res. 2011;346(2):255-62. https://doi.org/10.1007/s00441-011-1258-7.

42. Contaldo C, Högger DC, Khorrami Borozadi M, Stotz M, Platz U, Forster N, et al. Radial pressure waves mediate apoptosis and functional angiogenesis during wound repair in ApoE deficient mice. Microvasc Res. 2012;84(1):24-33. https://doi.org/10.1016/j.mvr.2012.03.006.

43. Kisch T, Wuerfel W, Forstmeier V, Liodaki E, Stang FH, Knobloch K, et al. Repetitive shock wave therapy improves muscular microcirculation. J Surg Res. 2016;201(2):440-5. https://doi.org/10.1016/j.jss.2015.11.049.

44. Reurink G, Goudswaard GJ, Moen MH, Weir A, Verhaar JA, Bierma-Zeinstra SM, Maas M, Tol JL; Dutch Hamstring Injection Therapy (HIT) Study Investigators. Platelet-rich plasma injections in acute muscle injury. N Engl J Med. 2014;370(26):2546-2547. https://doi.org/10.1056/NEJMc1402340.

45. de Albornoz PM, Aicale R, Forriol F, Maffulli N. Cell therapies in tendon, ligament, and musculoskeletal system repair. Sports Med Arthrosc Rev. 2018;26(2):48-58. https://doi.org/10.1097/JSA.0000000000000192.

\section{Publisher's Note}

Springer Nature remains neutral with regard to jurisdictional claims in published maps and institutional affiliations.

$$
\begin{aligned}
& \text { Ready to submit your research? Choose BMC and benefit from: } \\
& \text { - fast, convenient online submission } \\
& \text { - thorough peer review by experienced researchers in your field } \\
& \text { - rapid publication on acceptance } \\
& \text { - support for research data, including large and complex data types } \\
& \text { - gold Open Access which fosters wider collaboration and increased citations } \\
& \text { - maximum visibility for your research: over } 100 \mathrm{M} \text { website views per year }
\end{aligned}
$$

At $\mathrm{BMC}$, research is always in progress.

Learn more biomedcentral.com/submissions 